\title{
Situação vacinal de adolescentes escolares contra o HPV e fatores associados
}

\author{
HPV vaccination status of school adolescents and associated factors
}

Situación de la vacunación contra la VPH de los adolescentes en edad escolar y factores asociados

\begin{abstract}
RESUMO
Objetivo: Analisar a situação vacinal contra o HPV de adolescentes escolares e seus fatores associados. Método: Trata-se de um estudo descritivo com abordagem quantitativa, realizado com 502 adolescentes de escolas da rede pública de ensino municipal e estadual, situadas no município de Picos no estado do Piauí, no ano de 2018 e com dados do DATASUS. Resultados: Dos adolescentes pesquisados, o sexo feminino apresentou maior acesso e maior cobertura vacinal anual a vacina contra o HPV, representado respectivamente por $74,91 \%$ e $46,20 \%$ dos escolares. No entanto, de acordo com as informações do DATASUS, referentes ao acesso dos adolescentes demostraram que os meninos tiveram um maior acesso à vacina representado por $20,04 \%$, e também uma maior cobertura vacinal anual representado por 8,97\%. Conclusão: 0 acesso e a cobertura vacinal para este imunobiológico apresentam-se bem abaixo do recomendado, demandando assim, novas ações com objetivo de aumentar a adesão desses adolescentes a vacina.
\end{abstract}

DESCRITORES: Vacinação; Adolescente; Papillomaviridae; Saúde Pública.

\section{ABSTRACT}

Objective: To analyze the HPV vaccination status of school adolescents and its associated factors. Method: This is a descriptive study with quantitative approach, carried through with 502 adolescents of schools of the municipal and state public school system, located in the city of Picos in the state of Piauí, in the year of 2018 and with data from DATASUS. Results: Of the adolescents researched, the female sex presented greater access and greater annual vaccination coverage against HPV, represented respectively by $74.91 \%$ and $46.20 \%$ of the school children. However, according to DATASUS information, regarding the access of adolescents showed that boys had greater access to the vaccine represented by $20.04 \%$, and also greater annual vaccination coverage represented by $8.97 \%$. Conclusion: The access and vaccination coverage for this immunobiological are well below the recommended, thus requiring new actions in order to increase the adherence of these adolescents to the vaccine.

DESCRIPTORS: Vaccination; Adolescent; Papillomaviridae; Public Health.

\section{RESUMEN}

Objetivo: Analizar el estado de la vacunación contra el VPH de los escolares y sus factores asociados. Método: Se trata de un estudio descriptivo con enfoque cuantitativo, realizado con 502 adolescentes de escuelas públicas municipales y estaduales, ubicadas en la ciudad de Picos en el estado de Piauí, en el año 2018 y con datos de DATASUS. Resultados: De los adolescentes encuestados, el sexo femenino presentaba un mayor acceso y una mayor cobertura anual de vacunación contra el VPH, representando respectivamente el $74,91 \%$ y el $46,20 \%$ de los escolares. Sin embargo, de acuerdo con la información de DATASUS, en lo que respecta al acceso de los adolescentes, se observa que los varones tienen un mayor acceso a la vacuna, que representa el 20,04\% y también una mayor cobertura de vacunación anual, que representa el 8,97\%. Conclusión: El acceso y la cobertura de vacunación de este inmunobiológico están muy por debajo de lo recomendado, por lo que se requieren nuevas medidas para aumentar la adhesión de estos adolescentes a la vacuna.

DESCRIPTORES: Vacunación; Adolescente; Papillomaviridae; Salud Pública.

RECEBIDO EM: 14/10/2020 APROVADO EM: 29/10/2020

\section{Brenda Moreira Loiola}

Acadêmica do curso de Enfermagem pela Universidade Federal do Piauí; Membro do Grupo de Pesquisa em Saúde Coletiva UFPI/CNPq (Picos-PI, Brasil).

ORCID: 0000-0001-7625-5075 


\section{Pallysson Paulo da Silva}

Acadêmico do curso de Enfermagem pela Universidade Federal do Piaúi. Membro do Grupo de Pesquisa em Saúde Coletiva UFPI/CNPq (Picos-PI, Brasil).

ORCID: 0000-0002-3650-5938

\section{Simone Barroso de Carvalho}

Enfermeira. Mestre em Saúde e Comunidade. Especialista em Enfermagem em Cuidado pré-natal, Gestão em Saúde e UTI. Plantonista do Hospital Regional Justino Luz (Picos-PI, Brasil).

ORCID:0000-0001-7428-8420

\section{Edina Araújo Rodrigues Oliveira}

Enfermeira. Mestre em Enfermagem. Professora Adjunto I do curso de Enfermagem da Universidade Federal do Piauí. Membro do Grupo de Pesquisa em Saúde Coletiva - UFPI/CNPq (Picos-PI, Brasil).

ORCID: 0000-0002-6352-4202

\section{Lany Leide de Castro Rocha Campelo}

Enfermeira. Mestre e Doutora em Ciências (EEUSP). Pesquisadora colaboradora do Grupo de Estudos em Enfermagem e Família e do Grupo de Pesquisa em Saúde Coletiva, Coordenadora do Programa de Extensão Mais Sorriso mais Saúde e coordenadora do Estágio Curricular I e II do curso de Enfermagem da UFPI.

ORCID: 0000-0002-1686-9312

\section{Luísa Helena de Oliveira Lima}

Enfermeira. Doutora em Enfermagem. Professora Associada da UFPI, Docente dos Programas de Pós-Graduação em Ciências e Saúde; Saúde e Comunidade e do Mestrado Profissional em Saúde da Família. Coordenadora do Grupo de Pesquisa em Saúde Coletiva UFPI/CNPq.

ORCID: 0000-0002-1890-8590

\section{INTRODUÇÃO}

$\mathbf{0}$ adolescente é um indivíduo susceptível à diversos problemas de saúde pública que existem na sociedade. Nessa fase da vida aparecem muitas dúvidas, desejos que quando realizados de maneira incorreta podem resultar em diversos acontecimentos como as Infecções Sexualmente Transmissíveis (IST), nelas merece realce o Papiloma Vírus Humano (HPV) que pode provocar tumores malignos, especialmente do câncer de colo do útero e do pênis. Diante dessas características o Ministério da Saúde, por meio do Programa Nacional de Imunizações, em 2014, ampliou o Calendário Nacional de Vacinação e introduziu a vacina contra o HPV no Sistema Único de Saúde (SUS) ${ }^{(1)}$.

A cada ano uma faixa etária é abordada para receber a vacina. Com o início em 2014, o público escolhido foram as adolescentes do sexo feminino na faixa etária de 11 a 13 anos. Por apresentarem maior vulnerabilidade e menor número, as meninas indígenas de 9 a 13 anos de idade foram incluídas na rotina de vacinação, na sua totalidade nesse mesmo ano. Em 2015 e 2016, foram vacinadas meninas de 9 a 11 anos, e em 2017 o MS ampliou para a faixa etária de 9 a 14 anos para as meninas e incluiu o público masculino com idade de 11 a 14 anos, 11 meses e 29 dias ${ }^{(2)}$.

No Brasil, em 2014 foram vacinados com a dose 1 da HPV quadrivalente, cerca de 5.373.536 adolescentes do sexo feminino de 9 a 13 anos, assim, o país alcançou a cobertura vacinal de $108,45 \%$. No Piauí foram imunizadas 87.377 , correspondendo a $99,19 \%$ de cobertura vacinal. $\mathrm{Na}$ cidade de Picos no referido ano, foram aplicadas 2.030 doses, correspondendo a $106,73 \%$ do público vacinado. Comparando à dose 2 , notou-se uma redução brusca na quantidade de doses ministradas e consequentemente na cobertura vacinal. No país foram aplicadas apenas 3.212.780 doses com cobertura vacinal equivalente a $64,59 \%$, diminuição a qual também foi notável no estado do Piauí e na cidade de Picos, sendo que o primeiro teve apenas um quantitativo de 41.931 doses aplicadas com cobertura vacinal de apenas $47,45 \%$ e a nível municipal, essa cobertura foi ain- da menor, correspondendo a 40,10\% com apenas 770 doses aplicadas ${ }^{(3)}$.

No ano de 2018 em âmbito nacional 4 milhões de meninas, ou seja , $41,8 \%$ de 9 a 14 anos completaram o esquema de vacinação e 911 mil ou seja 12,7\% meninos de 11 a 14 anos completaram o esquema da vacina. No Piauí a cobertura vacinal foi de $42,2 \%$ entre meninas de 9 a 14 anos e $39 \%$ entre os meninos de 12 e 13 anos, com a primeira dose ${ }^{(4)}$.

Diante dos dados mostrados, percebe-se a redução da cobertura vacinal a nível nacional, estadual e municipal, o que demonstra a redução da adesão dos adolescentes em relação a vacina $\mathrm{HPV}$ ao longo do tempo, pelo fato da vacina ter saído das escolas e só estar acessível nos postos de saúde, onde os horários são mais restritos, interferindo assim na cobertura. Além da dificuldade do acesso, é visto também que os jovens frequentam pouco o serviço de saúde, diferente da criança, que os pais se preocupam mais em levá-las ao serviço, e que além disso, essa baixa procura se explica pelo típico comportamento de onipotência juvenil. $\mathrm{O}$ adolescente por pensar que nada de errado 
irá acontecer só desperta quando vê um caso próximo a ele ou recebe o diagnóstico de uma verruga, por exemplo ${ }^{(5)}$.

Perante o exposto, a realização do estudo se justifica em virtude de o público adolescente ser mais vulnerável para a infecção pelo HPV, e por isso deve estar empoderada sobre a importância da vacinação e imunizada contra os vírus do HPV. Assim sendo, o estudo é de extrema relevância, visto que é necessário reduzir de forma contínua os índices de infecção por HPV, bem como as consequências que ele provoca.

Desta maneira, o objetivo dessa pesquisa foi analisar a situação vacinal contra o HPV de adolescentes escolares e seus fatores associados.

\section{METÓDO}

Trata-se de um estudo descritivo, com abordagem quantitativa, realizado com 502 adolescentes de escolas de ensino fundamental e médio da rede pública de ensino, situadas no município de Picos, estado do Piauí, e dados informatizados do SUS. A população do estudo foi composta por 2.828 escolares do sexo feminino de 9 a 14 anos, 11 meses e 29 dias e do sexo masculino de 11 a 14 anos, 11 meses e 29 dias. Para obtenção da amostra para coleta de campo, foi feito o cálculo pela fórmula para estudos transversais com população finita ${ }^{(6)}$. A partir dessa fórmula, identificou-se que a amostra mínima deveria ser constituída por 338 indivíduos, porém obteve-se uma amostra maior de 502 adolescentes. As variáveis do referido estudo foram coletadas a partir de um questionário estruturado, o qual aborda os dados sociodemográficos e de práticas relacionadas à vacinação contra o HPV. A coleta de dados foi realizada em algumas unidades escolares do município selecionadas a partir de um sorteio até obter a amostra. Com relação aos dados do DATASUS ${ }^{(7)}$, a estimativa da população de adolescentes residentes em Picos no ano de 2018 (ano da coleta da pesquisa de campo) foi baseada no número de nascidos vivos que estariam no ano de $2018 \mathrm{com}$ a idade alvo da vacina: 9 a 14 anos para me- ninas e 11 a 14 anos para meninos. Com relação as doses da vacina contra o HPV segundo o Sistema do Programa Nacional de Imunizações (SI-PNI), utilizou-se o registro das doses aplicadas e informadas no SI-PNI no ano de $2018 \mathrm{em}$ adolescentes de Picos, segundo faixa etária e idade indicada para vacina contra o HPV. Com esses dados coletados em campo e no SI-PNI foi possível obter o acesso a vacinação, um indicador que mede $\mathrm{o}$ acesso da população-alvo à vacinação, ou seja, se o adolescente tomou pelo menos uma dose da vacina, já que o recomendado para imunização contra HPV é duas doses. Para melhor entendimento o acesso é uma proporção na qual o numerador, é o número de primeiras doses tomadas da vacina contra o HPV aplicadas na população selecionada no ano em que está sendo realizada a avaliação. $\mathrm{O}$ denominador é o número da população alvo da população selecionada, nesta pesquisa foi considerado o ano de 2018. Ademais, pode-se obter outra medida com os dados coletados que é a cobertura vacinal anual, esse indicador mede quantos adolescentes conseguiram tomar as duas doses da vacina, ou seja, ficaram imunizados contra o HPV. A cobertura vacinal anual é uma proporção na qual o numerador, é o número de segundas doses tomadas da vacina contra o HPV aplicadas na população selecionada no ano em que está sendo realizada a avaliação. $\mathrm{O}$ denominador é o número da população alvo da população selecionada, nesta pesquisa foi considerado o ano de $2018^{(8)}$. Os dados coletados foram tabulados e analisados no programa estatístico Statistical Package for the Social Sciences (SPSS), versão 20.0, estes foram organizados em tabelas e analisados com base em frequência absoluta e relativa. No estudo foram respeitados os preceitos éticos dispostos na Resolução 466/2012 acerca de pesquisa com seres humanos ${ }^{(9)}$. Foi utilizado o Termo de Consentimento Livre e Esclarecido (TCLE) e Termo de Assentimento Livre e Esclarecido (TALE). O projeto de pesquisa foi aprovado pelo Comitê de Ética em Pesquisa da Universidade Federal do Piauí com o Certificado de Apresentação de Apreciação de Ética-CAAE, número 2.429.531.

\section{RESULTADOS}

As informações referentes a idade mostraram que prevaleceu as meninas de 11 anos $(22,1 \%)$ e meninos de 12 anos $(29,15 \%)$. Quanto ao local de nascimento dos participantes, a grande maioria é da cidade que foi realizada a pesquisa. De acordo com a ocupação, a maioria apenas estuda, sendo $89,8 \%$ das meninas e $81,4 \%$ dos meninos. De acordo com a renda, os maiores índices são de baixa renda, sendo $41,9 \%$ do sexo feminino e $45,7 \%$ do sexo masculino vivendo apenas com o Programa Bolsa Família.

\section{As informações referentes a idade}

mostraram que prevaleceu as

meninas de 11 anos

\section{$(22,1 \%)$ e meninos}

\section{de 12 anos (29,15\%).}

Em relação a prática de vacinação observou-se que esteve associada com o sexo, sendo que meninas tiveram quase 3 vezes mais chance de serem vacinadas contra o HPV quando comparadas aos meninos. Além disso, a média de idade entre o grupo de vacinados foi ligeiramente maior. Vale salientar que, para esta análise, considerou-se vacinados todos os indivíduos que tinham 2 doses da vacina registradas na caderneta de vacinação.

As informações referentes ao acesso mostraram que $74,91 \%$ dos escolares do 
sexo feminino tiveram maior acesso a vacina. Enquanto no geral, $69,92 \%$ dos escolares tiveram acesso a vacina. Os dados referentes a cobertura vacinal anual demostraram que a maior cobertura vacinal foi no sexo feminino representado por $46,20 \%$ dos escolares. No geral, foi evidenciado que menos da metade dos escolares (36,85\%) atingiram cobertura vacinal anual, ou seja, tinha as duas doses da vacina registradas no cartão de vacinação (Tabela 1 ).

De acordo com os dados do SI-PNI, a caracterização da situação vacinal dos adolescentes residentes em Picos, mostram que os meninos tiveram um maior acesso à vacina representado por $20,04 \%$. No grupo geral mostrou que menos de um quarto dos adolescentes tiveram acesso a vacina representado por $17,95 \%$. Os dados referentes a cobertura vacinal anual demostraram que a maior cobertura vacinal foi no sexo masculino representado por $8,97 \%$. No geral, evidenciou-se que $8,26 \%$ dos adolescentes atingiram a cobertura vacinal anual (Tabela 2).

Outra variável analisada foi em relação aos motivos para a não vacinação dos escolares pesquisados, observou-se que os que prevaleceram foram: que não estou doente $(20,50 \%)$, que não gosta (19,87\%) e medo (14,83\%).

\section{DISCUSSÃO}

Os resultados apresentados na pesquisa informam que prevaleceu as meninas de 11 anos (22,1\%) e meninos de 12 anos
(29,15\%), percebe-se que é nessa idade onde geralmente ambos os sexos passam pela puberdade e se interessam mais por assuntos relacionados a sexualidade.

Os resultados mostraram que a prática de vacinação esteve associada com o sexo, sendo que meninas tiveram quase 3 vezes mais chance de serem vacinadas contra o HPV quando comparadas aos meninos. Além disso, a média de idade entre o grupo de vacinados foi ligeiramente maior, o mesmo verificou-se em um estudo transversal unicêntrico, feito com 390 escolares (idades entre 12 e 17 anos), onde $25,3 \%$ dos adolescentes afirmaram terem sido vacinados por ao menos uma dose. Entre eles, 10 eram do sexo masculino e os 89 restantes eram do sexo feminino. $\mathrm{O}$ que afirma que mais adolescentes do sexo feminino foram vacinadas contra o HPV do que do sexo masculino ${ }^{(10)}$.

O fato do grupo feminino vacinado ter uma idade maior que o masculino retratou também em um estudo que os pais possuíam uma crença de que a idade que pode iniciar a vacinação é precoce, pois a vacina protege contra uma IST, e eles acreditam que nessa idade suas filhas ainda não iniciaram a vida sexual, optando assim por levarem as suas filhas para se vacinarem com uma idade maior, na percepção que com uma menor idade o risco é menor de contrair uma IST, sendo que o Ministério da Saúde recomenda a vacina para meninas entre 9 a 14 anos de idade para a vacinação, visto que nesta faixa etária é provável

Tabela 1 - Caracterização da situação vacinal dos escolares pesquisados. Picos, Piauí, Brasil, 2018. n=502.

$\begin{array}{lccc} & \text { Veminino } & \text { Masculino } & \text { Geral } \\ \text { Acesso } & 74,91 \% & 62,31 \% & 69,92 \% \\ \text { Cobertura vacinal anual } & 46,20 \% & 22,61 \% & 36,85 \% \\ \text { Fonte: dados da pesquisa. } & & & \end{array}$

Tabela 2 - Caracterização da situação vacinal dos adolescentes picoenses. Picos, Piauí, Brasil, 2018. n=6181.

\begin{tabular}{|c|c|c|c|}
\hline Variáveis & Feminino & Masculino & Geral \\
\hline Acesso & $16,50 \%$ & $20,04 \%$ & $17,95 \%$ \\
\hline $\begin{array}{l}\text { Cobertura vacinal anual } \\
\text { Fonte: DATASUS }\end{array}$ & $7,77 \%$ & $8,97 \%$ & $8,26 \%$ \\
\hline
\end{tabular}

que não tenham começado o início da atividade sexual ${ }^{(11)}$.

As informações referentes ao acesso geral em Picos, ou seja, a primeira dose com $17,95 \%$, foi maior que a cobertura vacinal representada por $8,26 \%$, o mesmo foi evidenciado em um estudo feito sobre cobertura vacinal contra o HPV em meninas e adolescentes no Brasil, por análise de coortes de nascimentos, no ano de 2017. Foi feita a estimativa da cobertura da vacina contra HPV em três coortes de meninas elegíveis para a $1^{\circ}$ e $2^{\circ}$ dose de vacina apresentou que para a maioria dos estados, na segunda dose, há um predomínio de baixa cobertura da vacina contra $\mathrm{HPV}^{(12)}$.

Diante dos dados citados e a busca na literatura evidenciou-se alguns fatores para a negação da vacina contra o HPV, sendo estes o medo dos efeitos adversos e a impossibilidade de ir até a unidade de saúde para vacinar-se. Aponta-se também a ausência de conhecimento sobre o vírus, formas de transmissão e possíveis complicações, desconhecimento sobre os benefícios da vacina e preocupação dos responsáveis com os efeitos adversos ${ }^{(13)}$.

As informações referentes aos motivos para a não vacinação dos escolares mencionados nos resultados dessa pesquisa mostraram que os motivos que mais prevaleceram foram o fato de não estarem doentes para tomarem a vacina (20,50\%), de que não gostam (19,87\%) e pelo medo (14,83\%).

Em outro estudo observacional transversal sobre motivos para recusa da vacina contra o HPV entre adolescentes de 11 a 14 anos no município de Maringá-PR, verificou que o desconhecimento sobre o vírus foi citado por apenas $7 \%$ das adolescentes como motivo para não se vacinarem, enquanto o desconhecimento da vacina impediu que $8 \%$ delas procurassem o benefício, e quase $14 \%$ citaram a falta de conhecimento da campanha nacional de vacinação. Os motivos apontados com maior frequência foram o medo da injeção e dos efeitos colaterais ${ }^{(14)}$.

$\mathrm{O}$ fato dos motivos para não vacinação serem citados pela as duas pesquisas levarem ao mesmo motivo a falta de informação sobre vários fatores da vacinação 
reforçam a importância de ser realizado programas de educação e conscientização da população sobre o vírus e a vacina junto à campanha de imunização, a fim de se aumentar a adesão à vacina.

Portanto, é importante refletir essa temática da situação vacinal sob a ótica dos fatores associados a partir da fundamentação teórica acerca do cuidado e reconstrução das práticas de saúde, abordando a prevenção do câncer do colo do útero e as infecções causadas pelo HPV.

\section{CONCLUSÃO}

$\mathrm{O}$ acesso e a cobertura vacinal para este imunobiológico estão bem abaixo do reco- mendado, o que requer novas ações com objetivo de aumentar essa adesão entre os adolescentes. Vacinar com as duas doses no ambiente escolar parece ser uma boa estratégia para melhorar os níveis de cobertura vacinal contra o HPV.

A população da pesquisa teve em sua maioria meninas de idade maiores que meninos, nascidos em Picos, que apenas estudam e de baixa renda. Considerou-se que a prática de vacinação esteve associada com o sexo, sendo que meninas tiveram mais chance de serem vacinadas quando comparadas aos meninos e que os motivos para não vacinação que mais prevaleceram foram: que não estou doente, e que não gosta e pelo medo.
Diante disso, sugere-se a utilização de abordagens com base científica para incluir com mais efetividade os adolescentes nos serviços de saúde para prevenção do HPV na adolescência. O enfermeiro poderá desenvolver com base no estudo programas como palestras criativas e de fácil entendimento, sendo o foco as Unidades Básicas de Saúde (UBS), escolas, centros educacionais entre outros. É importante salientar a importância da equipe multidisciplinar para realizar uma busca ativa dos adolescentes não vacinados, e utilizar o Programa Saúde na Escola (PSE) para a imunização desses adolescentes, além de outras atividades de promoção e prevenção a saúde dessa população. m

\section{REFERÊNCIAS}

1. Mercante JIS, Rodrigues RG, Alvarenga MT, Kraievski ES. HPV e sua influência no câncer de colo de útero. Rev Conexão Eletrônica. 2017;14(1):182-189. Available from: http://revistaconexao. aems.edu.br/wp-content/plugins/download-attachments/includes/download.php?id=77

2. Ministério da Saúde (BR), Secretaria de Vigilância em Saúde, Coordenação-Geral do Programa Nacional de Imunizações. Informe técnico sobre a vacina papilomavírus humano (HPV) na atenção básica [Internet]. Brasília: Ministério da Saúde; 2014 [cited 2020 may 12]. Available from: https://portalarquivos2. saude.gov.br/images/pdf/2015/junho/26/Informe-T--cnico-Introdu----o-vacina-HPV-18-2-2014.pdf

3. Ministério da Saúde (BR), Secretaria de Vigilância em Saúde. Boletim Informativo do PNI-02/2016 - Vacinação contra HPV [Internet]. Brasilia: Ministério da Saúde; 2016 [cited 2020 may 06]. Available from: https:/www.conasems.org.br/wp-content/uploads/2016/10/ images_Boletim_informativo__HPV002-2016.pdf

4. Ministério da Saúde (BR). Ministério da Saúde convoca 10 milhões de adolescentes para vacinação de HPV e meningite [Internet]. Brasília: Ministério da Saúde; 2018 [cited 2020 may 06]. Available from: http://portalarquivos2.saude.gov.br/images/ pdf/2018/marco/13/Campanha-HPV-2018.pdf

5. Pinheiro C. O que afasta os jovens da vacina contra o HPV [Internet]. Veja Saúde; 2017 [cited 2019 set 19]. Available from: https://saude.abril.com.br/medicina/jovens-vacina-do-hpv/

6. Miot HA. Tamanho da amostra em estudos clínicos e experimentais. J Vasc Bras. 2011;10(4):275-278. Available from: https://www.scielo.br/pdf/jvb/v10n4/v10n4a01

7. Ministério da Saúde (BR), DATASUS, SIPNI - Sistema de Informações do Programa Nacional de Imunizações. Ministério da Saúde; 2018. Available from: <http://sipni-gestao.datasus.gov. br/si-pni-web/faces/relatorio/consolidado/dosesAplicadasCampanhaMultivacinacaoAdolescentes.jsf>.
8. Organização Pan-Americana da Saúde. Metodologia para o cálculo de cobertura da vacina contra o HPV na Região das Américas [Internet]. Washington, D.C.: OPAS; 2019. Available from: https://www.paho.org/pt/documents/methodology-calculate-hpv-vaccine-coverage-region-americas

9. Ministério da Saúde (BR), Conselho Nacional de Saúde. Resolução n 466, de 12 de dezembro de 2012 [Internet]. Brasília: Ministério da Saúde; 2012 [cited 2020 jul 01] Available from: http://conselho.saude.gov.br/resolucoes/2012/Reso466.pdf

10. Kreuger MRO, Lizott LS, Friedrich HA. Imunização contra HPV: nivel de conhecimento dos adolescentes. Adolesc Saude. 2017; 14(3):38-45 [cited 2020 jun 21]. Available from: https://cdn. publisher.gn1.link/adolescenciaesaude.com/pdf/v14n3a06.pdf

11. Reiter PL, Katz ML, Paskett ED. Correlates of HPV vaccination among adolescent

12. females from appalachia and reasons why their parents do not intend to vaccinate. Rev Vaccine. 2013;(31):3121-3125. Available from: doi:10.1016/j.vaccine.2013.04.068

13. Moura LL. Cobertura vacinal contra o Papilomavírus Humano (HPV) em meninas e adolescentes no Brasil: análise por coortes de nascimentos [dissertação]. Rio de Janeiro: Fundação Oswaldo Cruz, Escola Nacional de Saúde Pública Sergio Arouca; 2019.

14. Kornides ML, McRee AL, Gilkey MB. Parents who decline HPV vaccination: who later accepts and why? Acad Pediatr, review. 2018;18(2):37-43 [cited 2020 june 28]. DOI: https://dx.doi. org/10.1016\%2Fj.acap.2017.06.008

15. Zanini NV, Prado BS, Hendges RC, Santos CA, Rodovalho-Callegari FV, Bernuci MP. Motivos para recusa da vacina contra o Papilomavírus Humano entre adolescentes de 11 a 14 anos no município de Maringá-PR. Rev Bras Med Fam Comunidade. 2017;12(39):1-13. DOI: http://dx.doi.org/10.5712/rbmfc12(39) 1253 